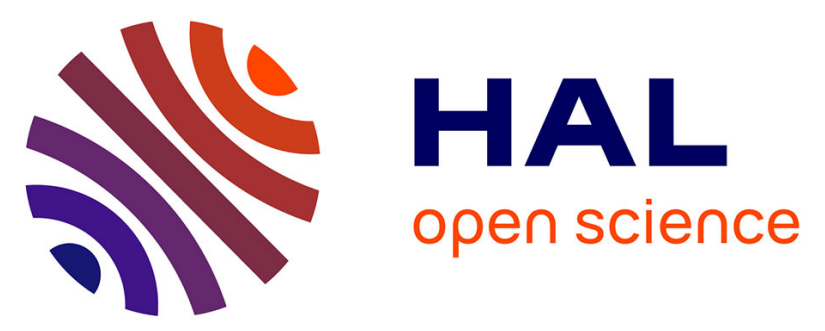

\title{
Évaluation de la cinétique globale et segmentaire du ventricule gauche chez les patients connus ou suspects de cardiopathie ischémique: comparaison des données apportées en scintigraphie de perfusion myocardique et en tomoventriculographie isotopique
}

A Halkovich, Denis Mariano-Goulart, M Sainmont, M Benkiran, Fayçal Ben Bouallègue

\section{- To cite this version:}

A Halkovich, Denis Mariano-Goulart, M Sainmont, M Benkiran, Fayçal Ben Bouallègue. Évaluation de la cinétique globale et segmentaire du ventricule gauche chez les patients connus ou suspects de cardiopathie ischémique: comparaison des données apportées en scintigraphie de perfusion myocardique et en tomoventriculographie isotopique. Médecine Nucléaire - Imagerie Fonctionnelle et Métabolique, 2014, 10.1016/j.mednuc.2013.12.006 . hal-02547819

\section{HAL Id: hal-02547819 \\ https://hal.umontpellier.fr/hal-02547819}

Submitted on 20 Apr 2020

HAL is a multi-disciplinary open access archive for the deposit and dissemination of scientific research documents, whether they are published or not. The documents may come from teaching and research institutions in France or abroad, or from public or private research centers.
L'archive ouverte pluridisciplinaire HAL, est destinée au dépôt et à la diffusion de documents scientifiques de niveau recherche, publiés ou non, émanant des établissements d'enseignement et de recherche français ou étrangers, des laboratoires publics ou privés. 


\title{
Évaluation de la cinétique globale et segmentaire du ventricule gauche chez les patients connus ou suspects de cardiopathie ischémique : comparaison des données apportées en scintigraphie de perfusion myocardique et en tomoventriculographie isotopique
}

\author{
Assessment of global and regional left ventricular contractile function in patients with known or \\ suspected coronary artery disease: Comparison between myocardial perfusion scintigraphy and \\ gated blood pool SPECT data
}

\author{
A. Halkovich *, D. Mariano-Goulart, A. Bourdon, M. Sainmont, M. Benkiran, F. Ben Bouallegue \\ Service de médecine nucléaire, CHRU Lapeyronie, 371, avenue du Doyen-Gaston-Giraud, 34295 Montpellier cedex 05, France
}

\begin{abstract}
Résumé
Objectifs. - L'analyse de la contraction ventriculaire gauche a une importance majeure dans le diagnostic et la prise en charge des patients atteints de cardiopathies. Le but de notre étude a été de comparer les paramètres étudiant la cinétique globale et segmentaire du ventricule gauche fournis par la tomoventriculographie isotopique et par la scintigraphie de perfusion myocardique.

Matériel et méthodes. - Deux groupes ont été constitués, le premier comprenant 23 scintigraphies de perfusion à la ${ }^{99 \mathrm{~m}} \mathrm{Tc}-\mathrm{Tetrofosmine}$ et le second 50 scintigraphies de perfusion au chlorure de ${ }^{201}$ Thallium, dont les données ont été comparées à celles de tomoventriculographies isotopiques réalisées de façon rapprochée.

Résultats. - Nous avons obtenu de bonnes corrélations ( $r=0,81$ à 0,94$)$ en ce qui concerne les paramètres fonctionnels globaux (fraction d'éjection et volumes télédiastolique et télésystolique) dans les deux groupes. D'assez bonnes corrélations existaient également à l'échelle segmentaire $(r=0,49$ à 0,62$)$, entre la fraction d'éjection segmentaire obtenue en tomoventriculographie et l'épaississement pariétal («Thickening ») ou le déplacement de la surface endocardique en systole (« Motion ») calculés en scintigraphie de perfusion. Ces corrélations étaient significativement plus faibles dans le groupe bilanté par scintigraphie au chlorure de ${ }^{201}$ Thallium, et ce d'autant plus pour les segments hypokinétiques.

Conclusion. - Bien que ces deux explorations scintigraphiques utilisent des approches très différentes, la tomoventriculographie isotopique et la scintigraphie de perfusion myocardique permettent une étude de la contraction ventriculaire gauche à l'échelle globale et segmentaire, avec des données bien corrélées mais restant néanmoins non interchangeables.
\end{abstract}

Mots clés : Scintigraphie de perfusion myocardique ; Tomoventriculographie isotopique ; Fraction d'éjection ventriculaire gauche ; Cinétique segmentaire du ventricule gauche ; Épaississement pariétal systolique myocardique

\begin{abstract}
Background and aims. - The analysis of the left ventricular contractile function plays a major role in the diagnosis and management of patients with cardiopathies. The aim of our study was to compare gated blood pool SPECT and myocardial perfusion scintigraphy for the assessment of the left ventricular wall contractility at the global and the segmental scales.

Material and methods. - The data of $23{ }^{99 \mathrm{~m}} \mathrm{Tc}$-Tetrofosmin perfusion scintigraphies, and $50{ }^{201}$ Thallium perfusion scintigraphies were compared to those of gated blood pool SPECT performed at close interval.
\end{abstract}

\footnotetext{
* Auteur correspondant.

Adressese-mail : aurore.halkovich@hotmail.fr (A. Halkovich), d-mariano_goulart@chu-montpellier.fr (D. Mariano-Goulart).
} 
Results. - The correlations were good ( $r=0.81$ to 0.94$)$ concerning the global parameters (left ventricular ejection fraction, end-diastolic and endsystolic volumes) in the two groups. Quite good correlations were also found at the segmental scale ( $r=0.49$ to 0.62$)$, between the segmental ejection fraction calculated in gated blood pool SPECT and the wall thickening or the wall motion estimated in perfusion scintigraphy. These correlations were significantly lower in the "201 Thallium perfusion scintigraphy" group than in the " $99 \mathrm{~m}$ Tc-Tetrofosmin perfusion scintigraphy" group, especially for hypokinetic segments.

Conclusion. - Although they use very different approaches, GBPS and MPS give data about global and segmental left ventricular wall contraction that are well correlated, but not strictly interchangeable.

Keywords: Myocardial perfusion scintigraphy; Gated blood pool SPECT; Left ventricular ejection fraction; Segmental myocardial kinetics; Myocardial wall thickening

\section{Introduction}

En cardiologie nucléaire, il est maintenant largement reconnu que l'évaluation des paramètres fonctionnels globaux (en particulier la fraction d'éjection ventriculaire gauche [1-3] et le volume télésystolique [3-5]) apporte des informations pronostiques majeures chez les patients connus ou suspects de coronaropathie [1-3,6], après un infarctus du myocarde [4], suivis pour insuffisance cardiaque [5], ou dans le cadre d'un bilan avant chirurgie vasculaire [7].

L'impact pronostique de l'analyse de la fonction contractile du ventricule gauche à l'échelle segmentaire a également été démontré [8-12], en plus de son rôle dans l'amélioration des performances diagnostiques de la scintigraphie de perfusion myocardique (SPM) [13-16], ou pour évaluer la viabilité myocardique $[17,18]$.

Bien que la scintigraphie de perfusion myocardique (SPM) et la tomoventriculographie isotopique (TVI) soient basées sur des hypothèses et des principes très différents, ces deux examens scintigraphiques permettent d'étudier la contraction ventriculaire gauche à l'échelle globale et segmentaire : la SPM étudie la fixation par la paroi myocardique du radiotraceur utilisé (complexes cationiques lipophiles marqués par le ${ }^{99 \mathrm{~m}}$ Technetium ou chlorure de ${ }^{201}$ Thallium), exploite l'effet de volume partiel pour estimer l'épaississement pariétal systolique (SPM-T) et utilise une segmentation basée sur une modélisation ellipsoïde pour estimer le déplacement de la surface endocardique en systole (SPM-M) [19-22]. En TVI, l'analyse est basée sur le marquage du pool sanguin circulant et l'évaluation des variations d'activité intraventriculaire gauche au cours du cycle cardiaque, corrélées aux variations de volume sanguin intracavitaire et ainsi à la fraction d'éjection globale et segmentaire [23-26].

La SPM et la TVI ont été comparées à l'échographie [27-29], à l'imagerie cardiaque par résonance magnétique (IRMc) [29-31], la ventriculographie par injection de produit de contraste iodé [32] et l'une avec l'autre [23,33] en ce qui concerne l'évaluation des paramètres fonctionnels globaux, avec de bonnes corrélations entre les résultats de ces différentes techniques.

Plusieurs études ont également montré de bonnes corrélations entre la SPM et l'IRMc [30,34], la SPM et l'échographie $[27,35]$, et entre la TVI et l'IRMc [23,24] pour l'évaluation de la fonction contractile segmentaire du ventricule gauche, et entre la SPM et la TVI pour la recherche d'asynchronisme intraventriculaire [23].
Les objectifs de cette étude étaient de comparer les paramètres étudiant la cinétique ventriculaire gauche globale et segmentaire obtenus en TVI en utilisant le logiciel de posttraitement Tompool ${ }^{\circledR}$ développé par notre équipe et déjà validé par plusieurs études, et en SPM à la ${ }^{99 \mathrm{~m}}$ Tc-Tetrofosmine ou au chlorure de ${ }^{201}$ Thallium, ceci tant pour des zones myocardiques normokinétiques que des zones avec une cinétique altérée, nécrosées ou sévèrement ischémiques, et présentant donc une faible fixation en SPM.

\section{Matériel et méthodes}

\subsection{Populations de patients}

Nous avons inclus de manière rétrospective l'ensemble des patients avec maladie coronaire connue ou suspectée, adressés dans notre service du CHU de Montpellier entre janvier 2004 et janvier 2013, chez qui avaient été réalisées à la fois une acquisition au repos de scintigraphie de perfusion myocardique à la ${ }^{99 \mathrm{~m}}$ Tc-Tetrofosmine ou au chlorure de ${ }^{201}$ Thallium et une tomoventriculographie isotopique, avec un délai maximal entre la réalisation des deux examens de 7 mois. La survenue d'un évènement cardiovasculaire intercurrent ou un changement de prise en charge thérapeutique durant cet intervalle constituait un critère de non-inclusion.

\subsubsection{Patients explorés par SPM à la ${ }^{99 m}$ Tc-Tetrofosmine}

Vingt-trois SPM à la ${ }^{99 \mathrm{~m}}$ Tc-Tetrofosmine ont été comparées à 23 TVI (19 patients masculins et 4 femmes ; âge : $64 \pm 7$ ans, minimum-maximum : 54-77 ans). Le délai entre la réalisation de la SPM et de la TVI était de 1,9 $\pm 2,2$ mois (minimummaximum : 0-7 mois). Le motif de réalisation de ces examens était : suivi de maladie coronaire $(n=16)$, bilan étiologique et fonctionnel de cardiomyopathie dilatée $(n=5)$, exploration de douleurs thoraciques atypiques $(n=1)$, bilan de dyspnée d'effort $(n=1)$.

Treize patients $(56,5 \%)$ avaient des séquelles de nécrose myocardique, localisées au niveau : apical $(n=2)$, de la paroi antérieure et de l'apex $(n=3)$, des parois antérieure, septale et apicale $(n=1)$, de la paroi inférieure et de l'apex $(n=4)$, des parois latérale, inférieure et apicale $(n=3)$. L'étendue des séquelles de nécrose (évaluée en SPM) était de $12 \pm 13 \%$ (minimum-maximum : 10-45\%). 
Deux patients étaient porteurs d'un pacemaker et trois d'un défibrillateur automatique implantable. Un patient présentait une insuffisance mitrale sévère (grade 3 ), trois une insuffisance mitrale minime à modérée (grade 1-2) et un patient avait une insuffisance aortique modérée (grade 2).

\subsubsection{Patients explorés par SPM au chlorure de ${ }^{201}$ Thallium}

Cinquante SPM au chlorure de ${ }^{201}$ Thallium ont été comparées à 50 TVI (41 patients masculins et 9 femmes, âge : $62 \pm 10$ ans, minimum-maximum : 40-76 ans). Le délai entre la réalisation de la SPM et de la TVI était de $0,16 \pm 0,71$ mois (minimum-maximum : 0-4 mois).

Tous les patients étaient adressés pour recherche de viabilité myocardique ou suivis après infarctus du myocarde. Quatre patients n'avaient pas de séquelle de nécrose. Chez les autres patients, les zones infarcies étaient localisées au niveau de la paroi antérieure et de l'apex $(n=14)$, des parois antérieure, septale et apicale $(n=25)$, antérieure, septale, inférieure et apicale $(n=4)$, antérieure, septale, latérale et apicale $(n=1)$ ou encore des parois antérieure, septale, latérale, inférieure et apicale $(n=2)$. L'étendue des séquelles de nécrose (évaluée en SPM) était de $36 \pm 19 \%$ (minimum-maximum : 10-75\%).

Onze patients étaient porteurs d'un défibrillateur automatique implantable. Un patient présentait une insuffisance mitrale sévère (grade 3) et sept avaient une insuffisance mitrale minime à modérée (grade 1-2).

\subsection{Acquisition des données de tomoventriculographie isotopique}

Les patients étaient maintenus au repos durant 15 minutes avant injection intraveineuse de $800 \mathrm{MBq}$ d'une solution d'érythrocytes autologues radiomarqués in vitro.

Les données étaient acquises à l'aide d'une gamma-caméra double tête (Infinia Hawkeye 1; GE Healthcare), têtes positionnées à $90^{\circ}$, avec des collimateurs parallèles basse énergie - haute résolution, selon les paramètres suivants : $6^{\circ}$ par pas sur $180^{\circ}$ (15 pas sur $90^{\circ}$ par tête), 40 secondes par pas, huit intervalles par cycle cardiaque avec une fenêtre de $10 \%$ du R$\mathrm{R}$, fenêtre spectrométrique de $10 \%$ autour du pic énergétique du ${ }^{99 m}$ Technetium $(140 \mathrm{keV} \pm 10 \%)$, matrice de $64 \times 64$ (taille de pixel : 5,9-6,8 mm), en accord avec les recommandations américaines [36] et européennes [37]. La durée totale des acquisitions était ainsi de l'ordre de 10 minutes.

\subsection{Post-traitement des acquisitions de tomoventriculographie isotopique}

Les acquisitions ont été reconstruites sur une station de travail Xeleris 2 (GE Healthcare, Chalfont St. Giles, RoyaumeUni), selon un algorithme OSEM (ordered subsets-expectation maximization) avec 2 itérations et 10 sous-ensembles, et en appliquant un filtre de Butterworth de fréquence 0,25 et d'ordre 10. Une correction du diffusé par méthode de Jaszczak [38] était également utilisée.
Les coupes transverses reconstruites étaient alors réorientées selon les axes cardiaques usuels, puis traitées grâce au logiciel de post-traitement semi-automatique développé dans notre service, Tompool ${ }^{\circledR}$ (téléchargeable gratuitement sur le net à http://scinti.etud.univ-montp1.fr/recherche/logiciels-a-telecharger/).

La dernière version de ce logiciel, qui a déjà été validée par plusieurs études [31,39], a été discrètement modifiée visà-vis de la version originale [25,40] : l'algorithme utilisé repose toujours sur le principe de ligne de partage des eaux, mais l'utilisation d'amincissements homotopiques successifs [25] a été remplacée par une approche d'immersion 3D prenant en compte les données à la fois de la coupe traitée et des coupes sus- et sous-jacentes, permettant de limiter le risque de sur-segmentation des cavités ventriculaires. Cette nouvelle version du logiciel a également été adaptée pour fonctionner sur les ordinateurs personnels usuels utilisant les différentes versions de Windows (Microsoft Corp., Redmond, WA).

Afin d'identifier chaque structure segmentée comme correspondant au ventricule gauche, au ventricule droit ou aux structures vasculaires en rapport avec les cavités ventriculaires, une première étape de définition des plans septal, des valves auriculo-ventriculaires et de l'infundibulum pulmonaire est réalisée de façon automatique par le logiciel, puis validée ou éventuellement modifiée si nécessaire par le médecin nucléaire.

Les courbes temps-activité globales comme locales sont obtenues à partir de la déformation d'une courbe de référence, tel que décrit par Caderas de Kerleau et al. [26], prenant en compte les données des 8 intervalles temporels subdivisant le cycle cardiaque. Pour chaque voxel de la surface ventriculaire gauche ou droite, l'activité correspondante est calculée selon la somme de l'activité de chaque voxel situé sur un rayon tracé entre la surface ventriculaire et le centre de masse du ventricule défini en télédiastole, tel que décrit par Vilain et al. [41].

La fraction d'éjection locale, à l'échelle de chaque voxel de la surface ventriculaire, est dérivée de chaque courbe tempsactivité locale après soustraction du bruit comme précédemment décrit [42] et les déviations standard de ces valeurs obtenues sont calculées. Ces valeurs ponctuelles de fraction d'éjection sont moyennées afin d'obtenir des valeurs segmentaires de fraction d'éjection (TVI-FEs), selon la cartographie à 20 segments usuellement utilisée [43] (Fig. 1).

La fraction d'éjection globale du ventricule gauche (FEVG), ainsi que les volumes télédiastolique (VTD) et télésystolique (VTS) du ventricule gauche sont calculés comme suit :

$$
F E V G=\frac{C_{E D}-C_{E S}}{C_{E D}} \text { et } V T D=\frac{C_{E D}}{C_{\max }} \cdot V_{\text {voxel }} \text { et } V T S=\frac{C_{E S}}{C_{\max }} \cdot V_{\text {voxel }}
$$

avec $\mathrm{C}_{\mathrm{ED}}$ et $\mathrm{C}_{\mathrm{ES}}$, le nombre de coups total au sein de la cavité ventriculaire gauche en fin de diastole et en fin de systole, respectivement. $\mathrm{C}_{\max }$, le nombre de coups maximal dans un voxel du ventricule gauche sur les huit différents intervalles de temps du cycle cardiaque et $\mathrm{V}_{\text {voxel }}$ le volume d'un voxel. 


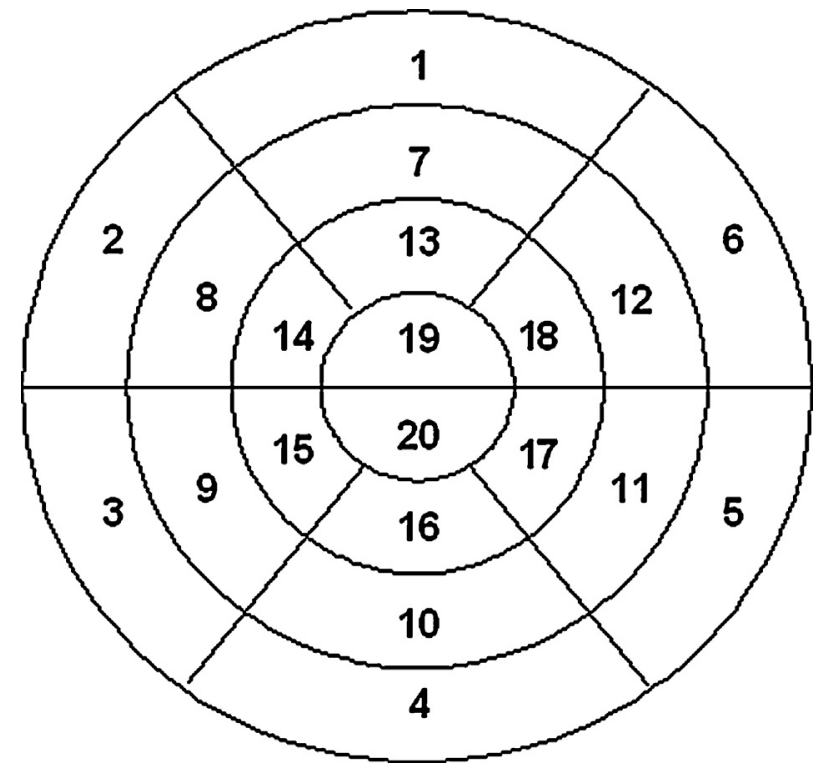

Fig. 1. Représentation sur une carte polaire de la subdivision du ventricule gauche en 20 segments.

Segmentation of the left ventricle using a 20-segment polar map.

Les mêmes calculs peuvent être appliqués au ventricule droit.

\subsection{Acquisition des données de scintigraphie de perfusion myocardique}

\subsubsection{Acquisitions de repos à la ${ }^{99 m}$ Tc-Tetrofosmine}

Toutes les acquisitions de SPM au repos ont été réalisées 3 heures après une acquisition en post-effort, si cette dernière n'était pas considérée comme normale par le médecin nucléaire. Le patient était maintenu 15 minutes au repos avant injection intraveineuse de $11 \mathrm{MBq} / \mathrm{kg}$ de ${ }^{99 \mathrm{~m}} \mathrm{Tc}$-Tetrofosmine, et les acquisitions étaient réalisées 15 à 30 minutes plus tard, patient installé en décubitus ventral, avec une gamma-caméra double tête (Infinia Hawkeye 1; GE Healthcare), têtes positionnées à $90^{\circ}$, avec des collimateurs parallèles basse énergie - haute résolution, selon les paramètres suivants : $6^{\circ}$ par pas sur $180^{\circ}$ (15 pas sur $90^{\circ}$ par tête), 40 secondes par pas, huit intervalles par cycle cardiaque avec une fenêtre de $10 \%$ du $\mathrm{R}-\mathrm{R}$, fenêtre spectrométrique de $10 \%$ autour du pic énergétique $\mathrm{du}{ }^{99 \mathrm{~m}}$ Technetium $(140 \mathrm{keV} \pm 10 \%)$, matrice de $64 \times 64$ (taille de pixel : 5,9-6,8 $\mathrm{mm}$ ), en accord avec les recommandations américaines [36] et européennes [37]. La durée totale des acquisitions était ainsi de l'ordre de 10 à 12 minutes.

\subsubsection{Acquisitions de repos au chlorure de ${ }^{201}$ Thallium}

Une première acquisition au repos était réalisée immédiatement après injection intraveineuse de $2 \mathrm{MBq} / \mathrm{kg}$ de chlorure de ${ }^{201}$ Thallium, puis les images de redistribution, dont les données ont été exploitées dans notre étude, étaient acquises 4 heures plus tard. Les patients étaient maintenus 15 minutes au repos avant chaque acquisition. Comme pour la SPM à la ${ }^{99 \mathrm{~m}}$ Tc-Tetrofosmine, les acquisitions étaient réalisées après installation du patient en décubitus ventral, sur une gammacaméra double tête (Infinia Hawkeye 1; GE Healthcare), têtes positionnées à $90^{\circ}$, avec des collimateurs parallèles basse énergie - haute résolution, selon les paramètres suivants : $6^{\circ}$ par pas sur $180^{\circ}$ (15 pas sur $90^{\circ}$ par tête), 40 secondes par pas, huit intervalles par cycle cardiaque avec une fenêtre de $10 \%$ du $\mathrm{R}-\mathrm{R}$, fenêtre spectrométrique de $15 \%$ autour du pic énergétique de $70 \mathrm{keV}$ et de $10 \%$ autour du pic de $167 \mathrm{keV}$ $\mathrm{du}{ }^{201}$ Thallium, matrice de $64 \times 64$ (taille de pixel : 5,9$6,8 \mathrm{~mm}$ ). La durée totale des acquisitions était également de l'ordre de 10 à 12 minutes.

\subsection{Post-traitement des acquisitions de scintigraphie de perfusion myocardique}

Les acquisitions de SPM à la ${ }^{99 \mathrm{~m}} \mathrm{Tc}$-Tetrofosmine au repos, comme celles de redistribution des SPM au chlorure de ${ }^{201}$ Thallium, ont été reconstruites sur une station de travail Xeleris 2 (GE Healthcare, Chalfont St. Giles, Royaume-Uni), selon un algorithme de rétroprojection filtrée et en appliquant un filtre de Butterworth de fréquence 0,4 et d'ordre 10. Les coupes reconstruites étaient alors réorientées selon les axes cardiaques usuels, puis traitées à l'aide du logiciel QGS/QPS (Cedars Sinai Medical Center, Los Angeles, CA, États-Unis), permettant une analyse visuelle ainsi que le calcul de paramètres quantitatifs segmentaires décrivant la perfusion et la cinétique locale (en particulier, calcul de l'épaississement pariétal systolique segmentaire ou «Thickening » [SPM-T] et du déplacement de la surface endocardique en systole ou « Motion » [SPM-M]), comme décrit par Germano et al. [22], et selon la même cartographie à 20 segments que celle utilisée pour la TVI.

\subsection{Analyses statistiques}

Les données quantitatives ont été décrites en termes de moyenne \pm écart-type.

Les corrélations entre SPM et TVI pour les valeurs de FEVG, VTD et VTS ont été étudiées par analyse de régression linéaire (avec notamment calcul du coefficient de Pearson) et calcul du coefficient de corrélation des rangs de Spearman. La concordance des données a été évaluée par calcul du coefficient de concordance de Lin. Une analyse de type Bland et Altman a été utilisée pour l'étude de biais et le calcul des limites d'agrément à $95 \%$.

L'analyse de régression linéaire et le calcul du coefficient de corrélation de Spearman ont également été appliqués pour étudier les relations entre les paramètres fonctionnels quantitatifs segmentaires obtenus en SPM (SPM-T et SPMM) et en TVI (TVI-FEs).

La significativité des coefficients de corrélation calculés a été évaluée par détermination de leur valeur de $p$ à deux degrés de liberté, avec un seuil de 0,05 comme risque de première espèce. La significativité d'une éventuelle différence entre deux coefficients de corrélation pour un même groupe de patients (par exemple la différence entre le coefficient de Pearson étudiant la relation entre SPM-T et TVI-FEs dans le groupe SPM au ${ }^{201}$ Thallium et le coefficient de Pearson étudiant la 
relation entre SPM-M et TVI-FEs dans ce même groupe de patients) a été évaluée par $t$-test de Williams. Pour la différence entre coefficients de corrélation de groupes de patients différents (par exemple, recherche de différence entre le coefficient de Pearson étudiant la relation entre SPM-T et TVIFEs dans le groupe SPM au ${ }^{201}$ Thallium et le coefficient de Pearson étudiant la relation entre SPM-T et TVI-FEs dans le groupe SPM à la ${ }^{99 m}$ Tc-Tetrofosmine), c'est le $z$-test de Fisher qui a été appliqué. Les différences entre deux coefficients de corrélation étaient là encore considérées comme significatives pour une valeur de $p<0,05$.

\section{Résultats}

\subsection{Paramètres fonctionnels globaux (FEVG, VTD, VTS)}

Il existait de bonnes corrélations entre les valeurs de FEVG, VTD et VTS obtenues en SPM et TVI (Tableau 1). La concordance était plus modérée, en particulier dans le groupe SPM au chlorure de ${ }^{201}$ Thallium, ceci en raison, comme montré par l'analyse de Bland et Altman, d'une tendance de la SPM à sous-estimer la FEVG et à surestimer les volumes ventriculaires comparativement à la TVI, de façon plus marquée en SPM au ${ }^{201}$ Thallium.

Il n'existait pas de différence significative de corrélation des données de SPM et de TVI entre les groupes de patients qui avaient bénéficié d'une SPM au chlorure de ${ }^{201}$ Thallium et ceux qui avaient été bilantés par SPM à la ${ }^{99 \mathrm{~m}}$ Tc-Tetrofosmine (Tableau 1, différence entre les coefficients de Pearson : $p>0,05)$.

\subsection{Paramètres segmentaires}

Les paramètres fonctionnels segmentaires obtenus en SPM et TVI ont été comparés segment par segment pour chaque patient (par exemple, comparaison des valeurs de SPM-T du segment $n^{\circ} 4$ et de TVI-FEs du segment 4 pour un même patient). Ainsi, avec la cartographie à 20 segments utilisée, $23 \times 20=460$ segments ont été étudiés dans le groupe de patients bilantés en SPM à la ${ }^{99} \mathrm{~m}$ Tc-Tetrofosmine, et $50 \times$ $20=1000$ segments pour le groupe SPM au chlorure de ${ }^{201}$ Thallium.

Il existait de bonnes corrélations entre les valeurs de TVIFEs et de SPM-T, et de TVI-FEs avec les valeurs de SPM-M (Tableau 2, Fig. 2), toutefois significativement meilleures dans le groupe de patients ayant bénéficié d'une SPM à $1{ }^{99} \mathrm{~m}^{\mathrm{T}} \mathrm{T}$ Tetrofosmine que dans celui bilanté par SPM au chlorure de ${ }^{201}$ Thallium (Tableau 2, différence entre les coefficients de Pearson : $p<0,05)$.

On ne retrouvait, par ailleurs, pas de différence significative de corrélation des données entre les hommes et les femmes (Tableau 3, différence entre les coefficients de Pearson: $p>0,05)$.

Afin de tenter de comparer ces corrélations entre zones myocardiques normokinétiques et zones hypokinétiques, nous

Tableau 1

Comparaison entre scintigraphie de perfusion myocardique et tomoventriculographie isotopique pour l'estimation des paramètres fonctionnels globaux : analyse de régression linéaire, coefficient de corrélation des rangs de Spearman, coefficient de concordance de Lin et analyse de Bland-Altman.

Comparison between MPS and GBPS for assessment of global left ventricular function parameters: linear regression analysis, Spearman's coefficient, and Bland and Altman analysis data.

\begin{tabular}{|c|c|c|c|c|c|c|}
\hline & \multicolumn{2}{|l|}{ FEVG } & \multicolumn{2}{|l|}{ VTD } & \multicolumn{2}{|l|}{ VTS } \\
\hline & $\begin{array}{l}\mathrm{SPM} \\
{ }^{99 \mathrm{~m}} \mathrm{Tc} \text {-Tetrofosmine }\end{array}$ & $\begin{array}{l}\text { SPM } \\
{ }^{201} \text { Thallium }\end{array}$ & $\begin{array}{l}\mathrm{SPM} \\
{ }^{99 \mathrm{~m}} \mathrm{Tc} \text {-Tetrofosmine }\end{array}$ & $\begin{array}{l}\text { SPM } \\
{ }^{201} \text { Thallium }\end{array}$ & $\begin{array}{l}\mathrm{SPM} \\
{ }^{99 \mathrm{~m}} \mathrm{Tc} \text {-Tetrofosmine }\end{array}$ & $\begin{array}{l}\text { SPM } \\
{ }^{201} \text { Thallium }\end{array}$ \\
\hline \multicolumn{7}{|l|}{ Moyenne $\pm S D$} \\
\hline TVI & $50 \pm 17 \%$ & $38 \pm 16 \%$ & $121 \pm 50 \mathrm{~mL}$ & $127 \pm 42 \mathrm{~mL}$ & $66 \pm 44 \mathrm{~mL}$ & $83 \pm 44 \mathrm{~mL}$ \\
\hline SPM & $40 \pm 15 \%$ & $28 \pm 11 \%$ & $145 \pm 87 \mathrm{~mL}$ & $182 \pm 89 \mathrm{~mL}$ & $96 \pm 80 \mathrm{~mL}$ & $138 \pm 84 \mathrm{~mL}$ \\
\hline \multicolumn{7}{|l|}{ Analyse de régression linéaire } \\
\hline Pente & 0,79 & 0,60 & 1,41 & 1,89 & 1,57 & 1,79 \\
\hline Ordonnée à l'origine $\left(\mathrm{y}_{0}\right)$ & 0,40 & 4,96 & $-25,47$ & $-58,97$ & $-6,39$ & $-10,88$ \\
\hline $\mathrm{R}^{2}$ & 0,78 & 0,78 & 0,65 & 0,81 & 0,74 & 0,89 \\
\hline Coefficient de corrélation de Pearson $(r)$ & 0,89 & 0,88 & 0,81 & 0,90 & 0,86 & 0,94 \\
\hline $\begin{array}{l}\text { Significativité du coefficient de } \\
\text { Pearson (valeur de } p \text { ) }\end{array}$ & $<0,001$ & $<0,001$ & $<0,001$ & $<0,001$ & $<0,001$ & $<0,001$ \\
\hline $\begin{array}{l}\text { Significativité de la différence entre les } \\
\text { coefficients de Pearson (valeur de } p \text { ) }\end{array}$ & \multicolumn{2}{|c|}{ Non significative $(p=0,86)$} & \multicolumn{2}{|c|}{ Non significative $(p=0,20)$} & \multicolumn{2}{|c|}{ Non significative $(p=0,09)$} \\
\hline $\begin{array}{l}\text { Coefficient de corrélation des } \\
\text { rangs de Spearman }\end{array}$ & 0,91 & 0,87 & 0,76 & 0,85 & 0,85 & 0,91 \\
\hline Coefficient de concordance de Lin & 0,72 & 0,64 & 0,66 & 0,53 & 0,65 & 0,58 \\
\hline \multicolumn{7}{|l|}{ Analyse de Bland-Altman } \\
\hline Moyenne des différences (SPM - TVI) & $-10,3$ & $-10,2$ & 23,8 & 48,9 & 30,9 & 51,5 \\
\hline Déviation standard (SD) & 7,86 & 8,33 & 55,1 & 55,4 & 47,9 & 45,8 \\
\hline $\begin{array}{l}\text { Limites de confiance à } 95 \% \\
\text { (moyenne } \pm 2 \times \mathrm{SD} \text { ) }\end{array}$ & {$[-26,1 ; 5,4]$} & {$[-26,9 ; 6,4]$} & {$[-86,3 ; 133,9]$} & {$[-62,0 ; 159,8]$} & {$[-64,9 ; 126,8]$} & {$[-40 ; 143]$} \\
\hline
\end{tabular}

FEVG : fraction d'éjection globale du ventricule gauche ; VTD : volume télédiastolique ; VTS : volume télésystolique ; SPM : scintigraphie de perfusion myocardique ; TVI : tomoventriculographie isotopique. 
Tableau 2

Comparaison des paramètres segmentaires obtenus en tomoventriculographie isotopique et en scintigraphie de perfusion myocardique : analyse de régression linéaire et coefficient de corrélation des rangs de Spearman.

Comparison between GBPS and MPS at the segmental scale: linear regression analysis and Spearman's rank order correlation coefficient.

\begin{tabular}{|c|c|c|c|c|}
\hline & \multicolumn{2}{|l|}{ TVI-FEs versus SPM-T } & \multicolumn{2}{|l|}{ TVI-FEs versus SPM-M } \\
\hline & $\begin{array}{l}\mathrm{SPM} \\
{ }^{99 \mathrm{~m}} \mathrm{Tc} \text {-Tetrofosmine }\end{array}$ & $\begin{array}{l}\text { SPM } \\
{ }^{201} \text { Thallium }\end{array}$ & $\begin{array}{l}\text { SPM } \\
{ }^{99 m} \text { Tc-Tetrofosmine }\end{array}$ & $\begin{array}{l}\text { SPM } \\
{ }^{201} \text { Thallium }\end{array}$ \\
\hline \multicolumn{5}{|l|}{ Analyse de régression linéaire } \\
\hline Pente & 0,4724 & 0,296 & 0,091 & 0,0629 \\
\hline Ordonnée à l'origine $\left(\mathrm{y}_{0}\right)$ & $-4,6655$ & $-0,2031$ & $-0,4197$ & 0,2783 \\
\hline $\mathrm{R}^{2}$ & 0,38 & 0,27 & 0,35 & 0,24 \\
\hline Coefficient de Pearson $(r)$ & 0,62 & 0,52 & 0,59 & 0,49 \\
\hline $\begin{array}{l}\text { Significativité du coefficient de } \\
\text { Pearson (valeur de } p \text { ) }\end{array}$ & $<0,001$ & $<0,001$ & $<0,001$ & $<0,001$ \\
\hline $\begin{array}{l}\text { Significativité de la différence entre les } \\
\text { coefficients de Pearson (valeur de } p \text { ) }\end{array}$ & \multicolumn{2}{|l|}{ Significative $(p=0,01)$} & \multicolumn{2}{|l|}{ Significative $(p=0,01)$} \\
\hline Coefficient de corrélation des rangs de Spearman & 0,63 & 0,49 & 0,59 & 0,50 \\
\hline
\end{tabular}

SPM : scintigraphie de perfusion myocardique.

avons réalisé une analyse de régression linéaire en subdivisant les pools de segments étudiés en fonction de différentes valeurs seuil de TVI-FEs, SPM-T et SPM-M (Tableau 4). Ces valeurs seuils ont été déterminées selon des résultats et des bases de données issues de sujets normaux déjà publiés [23,44,45], et selon une base de données locale de SPM à la ${ }^{99 \mathrm{~m}} \mathrm{Tc}$ Tetrofosmine considérées comme normales, afin de distinguer au mieux les segments normokinétiques ou discrètement hypokinétiques des segments sévèrement hypokinétiques à akinétiques. Aucune différence significative entre les sous-groupes ainsi constitués n'a été retrouvée dans une première analyse (Tableau $4 \mathrm{a}$, différence entre les coefficients de Pearson : $p>0,05)$

Aucune différence significative entre les sous-groupes ainsi constitués n'a été retrouvée dans une première analyse (Tableau 4a, différence entre les coefficients de Pearson : $p>0,05)$. Nous avons effectué une seconde analyse (Tableau $4 \mathrm{~b}$ ) après exclusion des segments basaux qui sont connus pour avoir physiologiquement un faible épaississement pariétal systolique [45-48] (et également une fraction d'éjection systolique

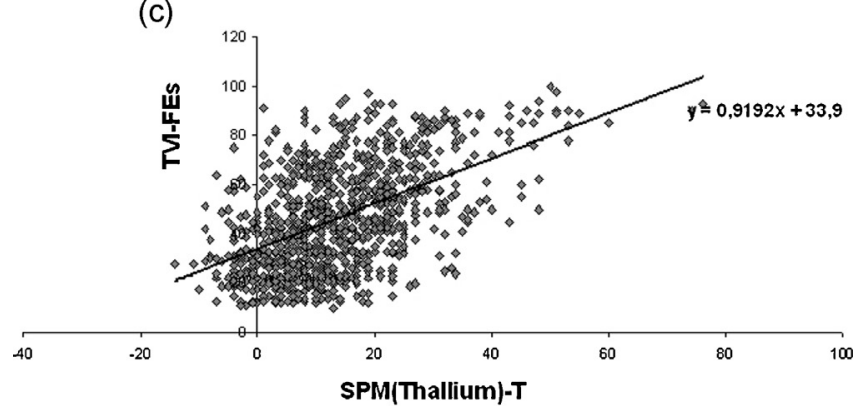

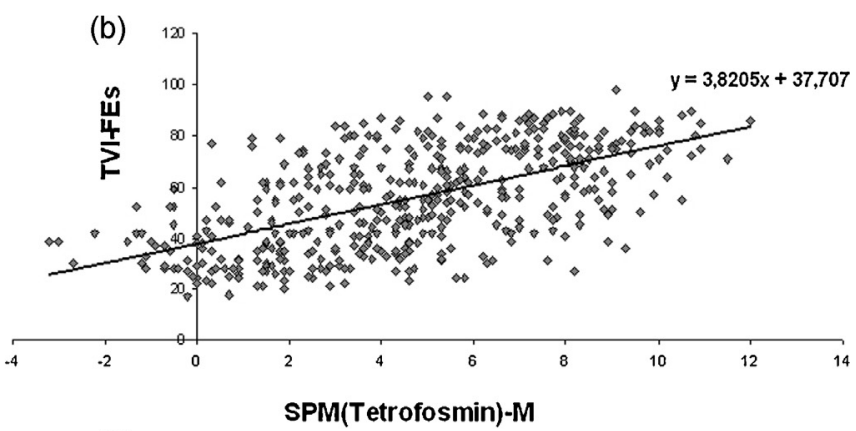

(c)

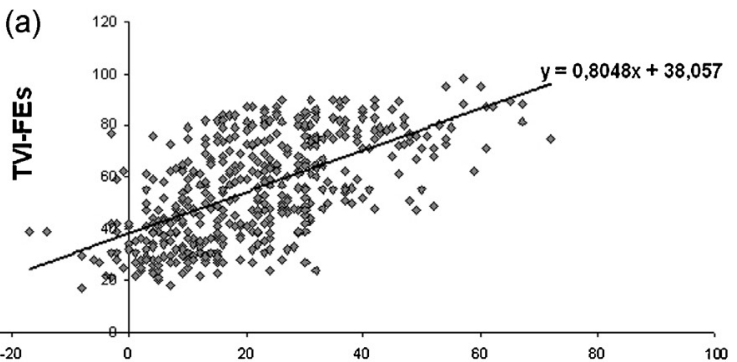

SPM(Tetrofosmin)-T

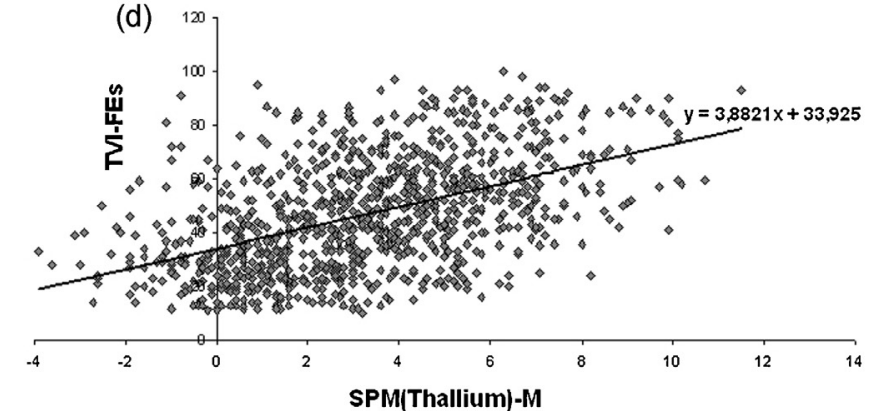

Fig. 2. Corrélations des paramètres segmentaires de tomoventriculographie isotopique (TVI) et scintigraphie de perfusion myocardique (SPM) : représentations graphiques et courbes de tendance $:$ a et $\mathrm{b}$ : groupe SPM à la ${ }^{99 \mathrm{~m}} \mathrm{Tc}$-Tetrofosmine : comparaison des données de TVI-FEs et SPM-T (a) et de TVI-FEs et SPM-M (b) ; $\mathrm{c}$ et $\mathrm{d}$ : groupe SPM au chlorure de ${ }^{201}$ Thallium : comparaison des données de TVI-FEs et SPM-T (c) et de TVI-FEs.

Correlations between GBPS segmental ejection fraction, and MPS segmental thickening and motion: data plots and trend lines: a and b: $99 m$ Tc-tetrofosmin MPS group: correlation between GBPS-sEF and MPS-T (a), and between GBPS-sEF and MPS-M (b) respectively; $c$ and d: ${ }^{201}$ Thallium MPS group: correlation between GBPS-sEF and MPS-T (c), and between GBPS-sEF and MPS-M (d) respectively. 
Tableau 3

Comparaison de la corrélation des données de tomoventriculographie isotopique et scintigraphie de perfusion myocardique chez les femmes et les hommes. Comparison of the correlation between GBPS and MPS for women and men.

\begin{tabular}{|c|c|c|c|c|c|c|}
\hline \multirow[t]{2}{*}{ Paramètres globaux } & \multicolumn{2}{|l|}{ FEVG } & \multicolumn{2}{|l|}{ VTD } & \multicolumn{2}{|l|}{ VTS } \\
\hline & $\begin{array}{l}\text { SPM } \\
{ }^{99 m} \text { Tc-Tetrofosmine }\end{array}$ & $\begin{array}{l}\text { SPM } \\
{ }^{201} \text { Thallium }\end{array}$ & $\begin{array}{l}\text { SPM } \\
{ }_{99 \mathrm{~m}} \mathrm{Tc} \text {-Tetrofosmine }\end{array}$ & $\begin{array}{l}\text { SPM } \\
{ }^{201} \text { Thallium }\end{array}$ & $\begin{array}{l}\text { SPM } \\
{ }_{99 \mathrm{~m}} \mathrm{Tc} \text {-Tetrofosmine }\end{array}$ & $\begin{array}{l}\text { SPM } \\
{ }^{201} \text { Thallium }\end{array}$ \\
\hline \multicolumn{7}{|l|}{ Femmes } \\
\hline Coefficient de Pearson $(r)$ & \multirow{3}{*}{$\begin{array}{l}0,88 \\
\mathrm{NS}(p=0,12) \\
0,8 \text { (NS) }\end{array}$} & 0,92 & 0,44 & \multirow{3}{*}{$\begin{array}{l}0,82 \\
<0,01 \\
0,75\end{array}$} & 0,79 & 0,92 \\
\hline Significativité de $r$ (valeur de $p$ ) & & $<0,001$ & NS $(p=0,56)$ & & NS $(p=0,21)$ & $<0,001$ \\
\hline Coefficient de Spearman & & 0,93 & 0,2 (NS) & & $0,4(\mathrm{NS})$ & 0,85 \\
\hline \multicolumn{7}{|l|}{ Hommes } \\
\hline Coefficient de Pearson $(r)$ & \multirow{3}{*}{$\begin{array}{l}0,93 \\
<0,001 \\
0,93\end{array}$} & 0,87 & 0,81 & \multirow{3}{*}{$\begin{array}{l}0,9 \\
<0,001 \\
0,86\end{array}$} & 0,87 & 0,94 \\
\hline Significativité de $r$ (valeur de $p$ ) & & $<0,001$ & $<0,001$ & & $<0,001$ & $<0,001$ \\
\hline Coefficient de Spearman & & 0,86 & 0,82 & & 0,93 & 0,92 \\
\hline $\begin{array}{l}\text { Significativité de la différence des } \\
\text { coefficients de Pearson chez les } \\
\text { femmes versus chez les hommes }\end{array}$ & \multicolumn{2}{|l|}{$\begin{array}{l}r \text { (femmes) } \\
\text { non significatif }\end{array}$} & $\begin{array}{l}r \text { (femmes) } \\
\text { non significatif }\end{array}$ & $\begin{array}{l}\text { NS } \\
(p=0,47)\end{array}$ & $\begin{array}{l}r \text { (femmes) } \\
\text { non significatif }\end{array}$ & $\begin{array}{l}\text { NS } \\
(p=0,73)\end{array}$ \\
\hline \multirow[t]{2}{*}{ Paramètres segmentaires } & \multicolumn{3}{|c|}{ TVI-FEs versus SPM-T } & \multicolumn{3}{|c|}{ TVI-FEs versus SPM-M } \\
\hline & \multicolumn{2}{|c|}{$\begin{array}{l}\text { SPM } \\
{ }^{99 \mathrm{~m}} \text { Tc-Tetrofosmine }\end{array}$} & $\begin{array}{l}\text { SPM } \\
{ }^{201} \text { Thallium }\end{array}$ & \multicolumn{2}{|c|}{$\begin{array}{l}\text { SPM } \\
{ }^{99 m} \text { Tc-Tetrofosmine }\end{array}$} & $\begin{array}{l}\text { SPM } \\
{ }^{201} \text { Thallium }\end{array}$ \\
\hline \multicolumn{7}{|l|}{ Femmes } \\
\hline Coefficient de Pearson $(r)$ & \multirow{3}{*}{\multicolumn{2}{|c|}{$\begin{array}{l}0,51 \\
<0,001 \\
0,52\end{array}$}} & 0,55 & \multirow{3}{*}{\multicolumn{2}{|c|}{$\begin{array}{l}0,55 \\
<0,001 \\
0,52\end{array}$}} & 0,46 \\
\hline Significativité de $r$ (valeur de $p$ ) & & & $<0,001$ & & & $<0,001$ \\
\hline Coefficient de Spearman & & & 0,52 & & & 0,47 \\
\hline \multicolumn{7}{|l|}{ Hommes } \\
\hline Coefficient de Pearson $(r)$ & \multirow{3}{*}{\multicolumn{2}{|c|}{$\begin{array}{l}0,65 \\
<0,001 \\
0,65\end{array}$}} & 0,50 & \multicolumn{2}{|l|}{0,61} & 0,50 \\
\hline Significativité de $r$ (valeur de $p$ ) & & & $<0,001$ & \multicolumn{2}{|c|}{$<0,001$} & $<0,001$ \\
\hline Coefficient de Spearman & & & 0,48 & 0,61 & & 0,51 \\
\hline Significativité de la différence des & \multicolumn{2}{|c|}{$\mathrm{NS}(p=0,09)$} & NS $(p=0,41)$ & \multicolumn{2}{|c|}{ NS $(p=0,47)$} & $\mathrm{NS}(p=0,53)$ \\
\hline
\end{tabular}

femmes versus chez les hommes

FEVG : fraction d'éjection globale du ventricule gauche ; VTD : volume télédiastolique ; VTS : volume télésystolique ; SPM : scintigraphie de perfusion myocardique ; TVI : tomoventriculographie isotopique.

segmentaire plus basse que les régions plus apicales $[44,46])$ et qui ne pouvaient donc être distingués de zones hypokinétiques pathologiques avec les valeurs seuils utilisées. Cette seconde analyse n'a pas non plus retrouvé de différence significative concernant les patients bilantés par SPM à la ${ }^{99 m} \mathrm{Tc}-$ Tetrofosmine. Par contre, pour les patients explorés par SPM au chlorure de ${ }^{201}$ Thallium, les données de SPM et de TVI étaient significativement moins bien corrélées pour les segments avec des valeurs basses de TVI-FEs $(<50 \%)$, de SPM-T $(<20 \%)$ et de SPM-M $(<3)$.

Nous avons ensuite regroupé les segments selon sept territoires anatomiques (Tableau 5) : antérieur, antéroseptal, inféroseptal, inférieur, inférolatéral, antérolatéral et apical. L'analyse a également été effectuée pour les segments basaux regroupés ensemble. Quelques hétérogénéités de force de corrélation entre les données de SPM et TVI ont ainsi été mises en évidence parmi ces différentes régions ventriculaires gauches. En particulier, la corrélation entre SPM-M et TVIFEs était plus basse au niveau des segments septaux que de la paroi latérale ou inférieure. Il n'existait pas de différence significative de corrélation entre SPM-T et TVI-FEs, sauf entre les segments inféroapicaux et l'apex, uniquement dans le groupe de patients explorés par SPM au chlorure de ${ }^{201}$ Thallium. Les données de TVI et de SPM n'étaient de plus que faiblement corrélées concernant les segments basaux dans ce même groupe de patients bilantés par SPM au chlorure de ${ }^{201}$ Thallium $(r=0,29$ et 0,25$)$.

\section{Discussion}

En ce qui concerne les paramètres fonctionnels globaux, les données de TVI et SPM étaient déjà connues pour être bien corrélées avec celles de l'IRM [24] ou de l'échographie cardiaque [27,28], et même l'une avec l'autre [33] (ceci n'avait toutefois pas encore été démontré avec l'utilisation du logiciel Tompool ${ }^{\mathbb{R}}$ ) et il n'était donc pas surprenant de retrouver un lien étroit des résultats de FEVG, VTD et VTS de ces deux examens dans notre étude. La tendance de la SPM à surestimer les volumes ventriculaires gauches et à sous-estimer la FEVG était également attendue, ceci étant déjà rapporté par Vanhove et al. [33], avec un biais d'autant plus marqué pour les volumes élevés. Ceci était également conforté par le fait qu'une telle surestimation des volumes et sous-estimation de la fraction d'éjection existait également entre la SPM et l'IRM cardiaque 
Tableau 4

Corrélation des paramètres segmentaires de tomoventriculographie isotopique et scintigraphie de perfusion myocardique en fonction de différentes valeurs seuil de fraction d'éjection segmentaire, d'épaississement pariétal systolique et de déplacement de la surface endocardique en systole.

Correlations between GBPS and MPS segmental parameters according to several thresholds of segmental ejection fraction, wall thickening and wall motion.

\begin{tabular}{|c|c|c|c|c|c|c|c|c|c|c|c|}
\hline \multirow{2}{*}{\multicolumn{2}{|c|}{$\begin{array}{l}\text { Corrélation entre } \\
\text { TVI-FEs et SPM-T }\end{array}$}} & \multicolumn{4}{|l|}{ TVI-FEs } & \multicolumn{6}{|l|}{ SPM-T } \\
\hline & & $<50 \%$ & $\geq 50 \%$ & $<30 \%$ & $\geq 30 \%$ & $<50 \%$ & $\geq 50 \%$ & $<30 \%$ & $\geq 30 \%$ & $<20 \%$ & $\geq 20 \%$ \\
\hline $\begin{array}{l}\text { SPM } \\
{ }^{99 m} \text { Tc-Ttfm }\end{array}$ & $\begin{array}{l}r \\
p_{(r)} \\
\text { Différence } \\
\text { entre les } r\end{array}$ & $\begin{array}{l}0,41 \\
<0,001 \\
\mathrm{NS} \\
(p=0,74)\end{array}$ & $\begin{array}{l}0,38 \\
<0,001\end{array}$ & $\begin{array}{l}0,21 \\
0,12 \\
\left(r_{\text {(FEs }<30}<3\right. \\
\text { significatif) }\end{array}$ & $\begin{array}{l}0,56 \\
<0,001 \\
\% \text { non }\end{array}$ & $\begin{array}{l}0,59 \\
<0,001 \\
\mathrm{NS} \\
(p=0,29\end{array}$ & $\begin{array}{l}0,41 \\
0,049\end{array}$ & $\begin{array}{l}0,47 \\
<0,001 \\
\mathrm{NS} \\
(p=0,08)\end{array}$ & $\begin{array}{l}0,32 \\
<0,001\end{array}$ & $\begin{array}{l}0,42 \\
<0,001 \\
\text { NS } \\
(p=0,76)\end{array}$ & $\begin{array}{l}0,39 \\
<0,001\end{array}$ \\
\hline $\begin{array}{l}\mathrm{SPM} \\
{ }^{201} \mathrm{~T} 1\end{array}$ & $\begin{array}{l}r \\
p_{(r)} \\
\text { Différence } \\
\text { entre les } r\end{array}$ & $\begin{array}{l}0,27 \\
<0,001 \\
\mathrm{NS} \\
(p=0,06)\end{array}$ & $\begin{array}{l}0,36 \\
<0,001\end{array}$ & $\begin{array}{l}0,04 \\
0,56 \\
\left(r_{(\text {FEs }<30}<\right. \\
\text { significatif) }\end{array}$ & $\begin{array}{l}0,47 \\
<0,001 \\
\% \text { non }\end{array}$ & $\begin{array}{l}0,49 \\
<0,001 \\
\left(r_{\text {(SPM-T }}\right. \\
\text { significat }\end{array}$ & $\begin{array}{l}0,01 \\
0,98 \\
0 \% \text { non }\end{array}$ & $\begin{array}{l}0,43 \\
<0,001 \\
\mathrm{NS} \\
(p=0,08)\end{array}$ & $\begin{array}{l}0,47 \\
<0,001\end{array}$ & $\begin{array}{l}0,29 \\
<0,001 \\
\mathrm{NS} \\
(p=0,06)\end{array}$ & $\begin{array}{l}0,41 \\
<0,001\end{array}$ \\
\hline
\end{tabular}

Corrélation entre

TVI-FEs

SPM-M

TVI-FEs et SPM-M

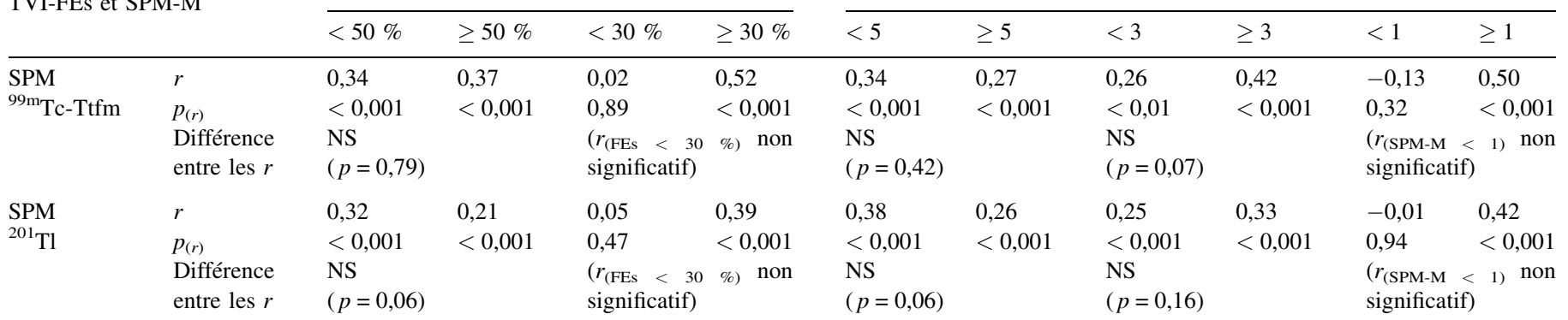

b. Analyse réalisée après exclusion des segments basaux

\begin{tabular}{|c|c|c|c|c|c|c|c|c|c|c|c|}
\hline \multicolumn{2}{|c|}{$\begin{array}{l}\text { Corrélation entre } \\
\text { TVI-FEs et SPM-T }\end{array}$} & \multicolumn{4}{|l|}{ TVI-FEs } & \multicolumn{6}{|l|}{ SPM-T } \\
\hline & & $<50 \%$ & $\geq 50 \%$ & $<30 \%$ & $\geq 30 \%$ & $<50 \%$ & $\geq 50 \%$ & $<30 \%$ & $\geq 30 \%$ & $<20 \%$ & $\geq 20 \%$ \\
\hline $\begin{array}{l}\text { SPM } \\
{ }^{99 m} \text { Tc-Ttfm }\end{array}$ & $\begin{array}{l}r \\
p_{(r)} \\
\text { Différence } \\
\text { entre les } r\end{array}$ & $\begin{array}{l}0,44 \\
<0,001 \\
\mathrm{NS} \\
(p=0,73)\end{array}$ & $\begin{array}{l}0,41 \\
<0,001\end{array}$ & $\begin{array}{l}0,13 \\
0,40 \\
\left(r_{\text {(FEs }<30}<\right. \\
\text { significatif) }\end{array}$ & $\begin{array}{l}0,64 \\
<0,001 \\
\% \text { non }\end{array}$ & $\begin{array}{l}0,68 \\
<0,001 \\
\left(r_{(\text {SPM-T }}\right. \\
\text { significa }\end{array}$ & $\begin{array}{l}0,41 \\
0,06 \\
0 \% \text { non }\end{array}$ & $\begin{array}{l}0,53 \\
<0,001 \\
\mathrm{NS} \\
(p=0,06)\end{array}$ & $\begin{array}{l}0,35 \\
<0,001\end{array}$ & $\begin{array}{l}0,47 \\
<0,001 \\
\mathrm{NS} \\
(p=0,82)\end{array}$ & $\begin{array}{l}0,49 \\
<0,001\end{array}$ \\
\hline $\begin{array}{l}\mathrm{SPM} \\
{ }^{201} \mathrm{Tl}\end{array}$ & $\begin{array}{l}r \\
p_{(r)} \\
\text { Différence } \\
\text { entre les } r\end{array}$ & $\begin{array}{l}0,23 \\
<0,001 \\
p<0,05\end{array}$ & $\begin{array}{l}0,53 \\
<0,001\end{array}$ & $\begin{array}{l}0,03 \\
0,67 \\
\left(r_{\text {(FEs }}<30\right. \\
\text { significatif })\end{array}$ & $\begin{array}{l}0,59 \\
<0,001 \\
\% \text { non }\end{array}$ & $\begin{array}{l}0,54 \\
<0,001 \\
\left(r_{\text {(SPM-T }}\right. \\
\text { significa }\end{array}$ & $\begin{array}{l}0,007 \\
0,98 \\
0 \% \text { non }\end{array}$ & $\begin{array}{l}0,43 \\
<0,001 \\
\mathrm{NS} \\
(p=0,64)\end{array}$ & $\begin{array}{l}0,48 \\
<0,001\end{array}$ & $\begin{array}{l}0,26 \\
<0,001 \\
p<0,05\end{array}$ & $\begin{array}{l}0,51 \\
<0,001\end{array}$ \\
\hline
\end{tabular}

Corrélation entre

TVI-FEs

SPM-M

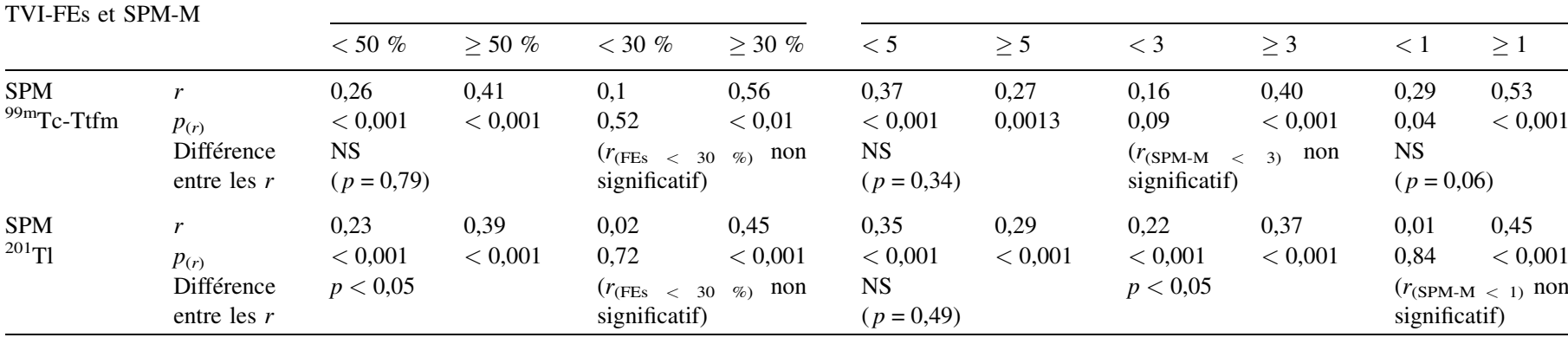

$r$ : coefficient de corrélation de Pearson $; p_{(r)}$ : valeur de $p$ du coefficient de Pearson (significativité) ; NS : différence entre les coefficients de Pearson non significative ; SPM : scintigraphie de perfusion myocardique ; TVI : tomoventriculographie isotopique.

[49], et que l'étude de Sibille et al. [31] montrait une discrète surestimation des volumes ventriculaires gauches par l'IRM comparativement à la TVI.

Une des limites de notre travail est que les examens de TVI et de SPM n'ont pas été réalisés « simultanément ». Néanmoins, plusieurs précautions ont été prises afin de limiter la variabilité de l'état physiologique du patient entre les deux acquisitions et de s'assurer de la comparabilité des données : les patients étaient gardés au repos au moins 15 minutes avant l'injection des radiotraceurs de SPM ou TVI, la majorité des examens ont 
Tableau 5

Analyse de corrélation après regroupement des segments en 7 territoires anatomiques (antérieur, antéroseptal, inféroseptal, inférieur, inférolatéral, antérolatéral et apical), ainsi que pour les segments basaux regroupés ensembles.

Correlation analysis after grouping the segments into seven anatomical regions (anterior, anteroseptal, inferoseptal, inferior, inferolateral, anterolateral, and apical regions). Analysis was also performed for basal segments taken together.

\begin{tabular}{|c|c|c|c|c|c|c|c|c|c|c|}
\hline & \multirow[b]{3}{*}{ Territoires } & \multirow[b]{2}{*}{ TVI-FEs } & \multirow{3}{*}{ SPM-T } & \multirow{3}{*}{ SPM-M } & \multicolumn{3}{|c|}{ TVI-FEs versus SPM-T } & \multicolumn{3}{|c|}{ TVI-FEs versus SPM-M } \\
\hline & & & & & \multicolumn{2}{|c|}{$\begin{array}{l}\text { Analyse de régres- } \\
\text { sion linéaire }\end{array}$} & \multirow[t]{2}{*}{$\mathrm{Sp}$} & \multicolumn{2}{|c|}{$\begin{array}{l}\text { Analyse de régres- } \\
\text { sion linéaire }\end{array}$} & \multirow[t]{2}{*}{$\mathrm{Sp}$} \\
\hline & & (Moyenn & & & $r$ & $p(r)$ & & $r$ & $p(r)$ & \\
\hline \multirow[t]{8}{*}{$\begin{array}{l}\text { SPM } \\
{ }^{99 m} \text { Tc-Ttfm }\end{array}$} & $\begin{array}{l}\text { Antérieur } \\
\text { (segments } 1,7 \text { et } 13 \text { ) }\end{array}$ & $54 \pm 19$ & $22 \pm 14$ & $6,3 \pm 2,6$ & 0,59 & $<0,001$ & 0,59 & 0,58 & $<0,001$ & 0,57 \\
\hline & $\begin{array}{l}\text { Antéroseptal } \\
\text { (segments } 2,8 \text { et } 14 \text { ) }\end{array}$ & $50 \pm 18$ & $19 \pm 12$ & $3,4 \pm 2,5$ & 0,64 & $<0,001$ & 0,61 & 0,38 & 0,001 & 0,42 \\
\hline & $\begin{array}{l}\text { Inféroseptal } \\
\text { (segments } 3,9 \text { et } 15 \text { ) }\end{array}$ & $54 \pm 20$ & $21 \pm 15$ & $2,9 \pm 2,1$ & 0,67 & $<0,001$ & 0,64 & 0,45 & $<0,001$ & 0,41 \\
\hline & $\begin{array}{l}\text { Inférieur } \\
\text { (segments } 4,10 \text { et } 16 \text { ) }\end{array}$ & $59 \pm 20$ & $19 \pm 15$ & $4,1 \pm 2,6$ & 0,64 & $<0,001$ & 0,61 & 0,73 & $<0,001$ & 0,70 \\
\hline & $\begin{array}{l}\text { Inférolatéral } \\
\text { (segments } 5,11 \text { et } 17 \text { ) }\end{array}$ & $59 \pm 20$ & $19 \pm 15$ & $5,5 \pm 3,0$ & 0,60 & $<0,001$ & 0,63 & 0,78 & $<0,001$ & 0,75 \\
\hline & $\begin{array}{l}\text { Antérolatéral } \\
\text { (segments } 6,12 \text { et } 18 \text { ) }\end{array}$ & $59 \pm 20$ & $24 \pm 14$ & $6,6 \pm 2,8$ & 0,56 & $<0,001$ & 0,56 & 0,74 & $<0,001$ & 0,72 \\
\hline & $\begin{array}{l}\text { Apex } \\
\text { (segments } 19 \text { et } 20 \text { ) }\end{array}$ & $50 \pm 19$ & $24 \pm 21$ & $3,0 \pm 3,2$ & 0,76 & $<0,001$ & 0,75 & 0,63 & $<0,001$ & 0,58 \\
\hline & $\begin{array}{l}\text { Segments basaux } \\
\text { (segments } 1 \text { à } 6)\end{array}$ & $57 \pm 17$ & $15 \pm 9$ & $5,3 \pm 2,9$ & 0,51 & $<0,001$ & 0,50 & 0,53 & $<0,001$ & 0,50 \\
\hline \multirow[t]{8}{*}{$\begin{array}{l}\mathrm{SPM} \\
{ }^{201} \mathrm{Tl}\end{array}$} & $\begin{array}{l}\text { Antérieur } \\
\text { (segments } 1,7 \text { et } 13 \text { ) }\end{array}$ & $43 \pm 21$ & $12 \pm 10$ & $3,6 \pm 2,7$ & 0,53 & $<0,001$ & 0,47 & 0,55 & $<0,001$ & 0,55 \\
\hline & $\begin{array}{l}\text { Antéroseptal } \\
\text { (segments } 2,8 \text { et } 14 \text { ) }\end{array}$ & $41 \pm 19$ & $11 \pm 11$ & $2,5 \pm 2,5$ & 0,54 & $<0,001$ & 0,52 & 0,37 & $<0,001$ & 0,35 \\
\hline & $\begin{array}{l}\text { Inféroseptal } \\
\text { (segments } 3,9 \text { et } 15 \text { ) }\end{array}$ & $50 \pm 19$ & $15 \pm 12$ & $2,9 \pm 2,3$ & 0,40 & $<0,001$ & 0,34 & 0,29 & $<0,001$ & 0,26 \\
\hline & $\begin{array}{l}\text { Inférieur } \\
\text { (segments } 4,10 \text { et } 16 \text { ) }\end{array}$ & $51 \pm 21$ & $15 \pm 12$ & $3,4 \pm 2,4$ & 0,49 & $<0,001$ & 0,46 & 0,56 & $<0,001$ & 0,59 \\
\hline & $\begin{array}{l}\text { Inférolatéral } \\
\text { (segments } 5,11 \text { et } 17 \text { ) }\end{array}$ & $51 \pm 21$ & $16 \pm 12$ & $3,7 \pm 2,7$ & 0,54 & $<0,001$ & 0,52 & 0,62 & $<0,001$ & 0,60 \\
\hline & $\begin{array}{l}\text { Antérolatéral } \\
\text { (segments } 6,12 \text { et } 18 \text { ) }\end{array}$ & $49 \pm 21$ & $14 \pm 10$ & $4,0 \pm 2,4$ & 0,53 & $<0,001$ & 0,50 & 0,50 & $<0,001$ & 0,51 \\
\hline & $\begin{array}{l}\text { Apex } \\
\text { (segments } 19 \text { et } 20)\end{array}$ & $36 \pm 15$ & $12 \pm 13$ & $1,8 \pm 2,6$ & 0,63 & $<0,001$ & 0,35 & 0,39 & $<0,001$ & 0,27 \\
\hline & $\begin{array}{l}\text { Segments basaux } \\
\text { (segments } 1 \text { à } 6)\end{array}$ & $57 \pm 16$ & $14 \pm 9,5$ & $4,5 \pm 2,4$ & 0,29 & $<0,001$ & 0,25 & 0,25 & $<0,001$ & 0,22 \\
\hline
\end{tabular}

SPM : scintigraphie de perfusion myocardique ; TVI : tomoventriculographie isotopique.

été réalisés de façon très rapprochée (groupe SPM à la ${ }^{99} \mathrm{~m}$ TcTetrofosmine : $52 \%$ des SPM ont été réalisées dans le même mois que la TVI et $78 \%$ dans les 2 mois, groupe SPM au chlorure de ${ }^{201}$ Thallium : $92 \%$ des SPM réalisées à moins de 5 jours d'écart de la TVI), et les patients avec survenue d'évènement cardiovasculaire ou changement de prise en charge thérapeutique entre la SPM et la TVI n'étaient pas inclus dans l'étude.

Concernant les résultats obtenus à l'échelle segmentaire, nous avons trouvé de relativement bonnes corrélations entre les données de TVI et SPM (Tableau 2), aussi bien entre le déplacement de la surface endocardique en systole et la fraction d'éjection segmentaire qu'entre l'épaississement pariétal systolique et la fraction d'éjection segmentaire.

Les données de SPM au chlorure de ${ }^{201}$ Thallium étaient significativement moins bien corrélées à la TVI-FEs que les données de SPM à la ${ }^{99 m}$ Tc-Tetrofosmine (Tableau 2, différence entre les coefficients de Pearson : $p<0,05)$. Une des explications potentielles est la moindre qualité d'image en scintigraphie au ${ }^{201}$ Thallium. Ceci découle d'abord de la plus faible activité injectée et fixée au myocarde (le thallium étant capté 3 fois plus que la tetrofosmine, mais l'activité injectée étant 5,5 fois plus faible afin de limiter l'irradiation du patient). La physique de détection des photons du ${ }^{201}$ Thallium est de plus moins avantageuse sur les gamma-caméras classiques que pour ceux du ${ }^{99}$ Technétium (plus de diffusé Compton et d'atténuation des photons de basse énergie du ${ }^{201}$ Thallium, détection moins efficace et moins résolue spatialement, avec un moins bon rapport signal sur bruit [50]).

En plus de cela, les patients bilantés par SPM au chlorure de ${ }^{201}$ Thallium avaient des séquelles de nécrose plus étendues et, globalement, des cardiopathies plus sévères que dans le groupe 
SPM à la ${ }^{99 m}$ Tc-Tetrofosmine, comme en témoignent la valeur moyenne de FEVG plus basse et les volumes plus élevés dans le groupe SPM au ${ }^{201}$ Thallium (Tableau 1). Or, en présence de tels défects perfusionels sévères et étendus, l'algorithme du logiciel QGS/QPS doit compenser ces zones en défaut de signal par application d'un modèle ellipsoïde pour relier les surfaces médioventriculaires des zones adjacentes bien perfusées $[19,22]$ et les surfaces endocardique et épicardique sont dérivées de ces résultats. La fiabilité de la SPM pour l'évaluation de l'épaississement systolique ou du déplacement de la surface endocardique en systole peut donc être sujette à caution dans ces zones « déduites » [51].

Ces hypothèses pourraient également expliquer les résultats obtenus en étudiant les corrélations des paramètres segmentaires en fonction de différentes valeurs seuils de TVI-FEs, SPM-T et SPM-M : dans le groupe SPM à la ${ }^{99 m} \mathrm{Tc}-$ Tetrofosmine, où les séquelles de nécrose myocardique étaient plus rares et de plus petite taille, aucune différence significative de corrélation n'a été retrouvée entre les segments considérés comme normokinétiques et les segments sévèrement hypokinétiques à akinétiques, ce qui souligne la fiabilité des résultats obtenus, même dans les zones sévèrement ischémiques ou nécrosées présentant un faible signal en SPM, si une délinéation correcte de la paroi ventriculaire est effectuée au préalable. Il est néanmoins à signaler que les données recueillies étaient insuffisantes pour atteindre la significativité en analyse de régression linéaire pour les sous-groupes TVI-sFE $<30 \%$ et $\mathrm{SPM}-\mathrm{M}<1$.

A contrario, dans le groupe SPM au chlorure de ${ }^{201}$ Thallium, où les patients présentaient des séquelles de nécrose plus étendues, et après avoir exclus de l'analyse les segments basaux qui ont physiologiquement des valeurs basses de SPM-T [45-48] (et à moindre mesure de fraction d'éjection segmentaire $[44,46])$, nous avons trouvé des corrélations significativement moins bonnes entre SPM et TVI pour les segments avec les valeurs basses de TVI-FEs, SPM-T et SPM$M$, avec une potentielle implication de problèmes de segmentation de la paroi myocardique dans ces larges zones nécrotiques très hypokinétiques nécessitant une compensation par fitting ellipsoïde.

Avoir basé notre analyse sur de telles valeurs seuils de fraction d'éjection segmentaire, d'épaississement pariétal et de déplacement de la surface endocardique, plutôt que d'avoir sélectionné les segments ischémiques et nécrotiques en fonction de leur score de perfusion en SPM, avait plusieurs avantages : cela nous affranchissait du problème d'autoatténuation à l'origine d'hypofixations artéfactuelles de segments normoperfusés, écartait également le risque de perfusion apparaissant faussement normale chez un patient tritronculaire équilibré, nous assurait d'isoler spécifiquement les segments sévèrement ischémiques avec cinétique altérée et nous permettait également d'inclure dans l'analyse les segments nécrosés.

Concernant l'analyse regroupant les segments en territoires anatomiques, de nombreuses études ont décrit des hétérogénéités régionales physiologiques de fraction d'éjection segmentaire, d'épaississement pariétal et de déplacement de la surface endocardique, au sein de la paroi ventriculaire gauche. Ces variations sont tout particulièrement en rapport avec l'anatomie et la complexité du mouvement tridimensionnel effectué par le ventricule gauche lors de sa systole, associant un raccourcissement longitudinal, un raccourcissement circonférentiel et un mouvement de torsion le long de l'axe longitudinal. Il existe ainsi un gradient apico-basal pour les valeurs de fraction d'éjection segmentaire $[44,46]$ et d'épaississement pariétal systolique [45-48] et un gradient transverse pour le déplacement de la surface endocardique [45] (avec des valeurs plus basses au niveau du septum que de la paroi latérale). Il nous a ainsi paru intéressant de rechercher s'il existait des variations des corrélations de nos paramètres segmentaires parmi les différentes régions du ventricule gauche. Nous avons également regroupé les segments basaux entre eux, du fait de leur particularité à avoir physiologiquement de faibles valeurs d'épaississement pariétal systolique et de leur localisation au contact des structures valvulaires dont le fonctionnement impacte sur leur cinétique.

Dans nos populations avec des séquelles d'infarctus intéressant le plus souvent les segments apicaux (35\% des patients ayant eu une SPM à la ${ }^{99 \mathrm{~m}}$ Tc-Tetrofosmine, $86 \%$ des patients pour les SPM au chlorure de ${ }^{201}$ Thallium), les gradients apico-basaux physiologiques de fraction d'éjection segmentaire et d'épaississement pariétal étaient effacés, remplacés par de faibles valeurs apicales de ces paramètres.

La corrélation entre SPM-T et TVI-FEs était relativement stable au sein du ventricule gauche, alors qu'il existait une moins bonne corrélation entre SPM-M et TVI-FEs au niveau de la paroi septale (qui présente physiologiquement de faibles valeurs de déplacement de la surface endocardique en systole).

Ces différents résultats soulignent que même s'il existe de relativement bonnes corrélations entre ces paramètres segmentaires, la fraction d'éjection segmentaire n'est que partiellement reflétée par l'évaluation de l'épaississement pariétal ou le déplacement de la surface endocardique en systole.

\section{Conclusion}

De bonnes corrélations entre les données de tomoventriculographie isotopique et de scintigraphie de perfusion myocardique ont été retrouvées dans cette étude en ce qui concerne l'évaluation de la fraction d'éjection ventriculaire gauche et des volumes télédiastoliques et télésystoliques qui reflètent la cinétique globale du ventricule gauche. Les valeurs de concordance de ces paramètres étaient plus modérées, en particulier dans le groupe bilanté par scintigraphie de perfusion au chlorure de ${ }^{201}$ Thallium, du fait d'une tendance à la sousestimation de la fraction d'éjection et à la surestimation des volumes ventriculaires en scintigraphie de perfusion myocardique, ce qui rappelle la prudence à adopter pour intégrer ces résultats au sein de comptes rendus médicaux.

Nous avons également trouvé d'assez bonnes corrélations des paramètres fonctionnels segmentaires (fraction d'éjection segmentaire calculée en TVI, épaississement pariétal et déplacement de la surface endocardique en systole estimés en SPM), qui apparaissaient altérées en cas de larges séquelles 
de nécrose myocardique, avec une implication potentielle de difficultés de segmentation de la paroi myocardique par les logiciels de post-traitement des SPM dans de telles circonstances.

À l'échelle globale et segmentaire, la tomoventriculographie isotopique apporte ainsi des informations complémentaires aux données de scintigraphie myocardique pour l'analyse fonctionnelle du ventricule gauche, avec une valeur ajoutée d'autant grande lorsqu' une recherche d'asynchronisme, ou une étude du ventricule droit sont également d'intérêt pour le patient.

\section{Déclaration d'intérêts}

Les auteurs déclarent ne pas avoir de conflits d'intérêts en relation avec cet article.

\section{Annexe A. Matériel complémentaire}

Le matériel complémentaire (Fig. E1, E2 et tableau E1) accompagnant la version en ligne de cet article est disponible sur

\section{Références}

[1] Sharir T, Germano G, Kang X, Lewin HC, Miranda R, Cohen I, et al. Prediction of myocardial infarction versus cardiac death by gated myocardial perfusion SPECT: risk stratification by the amount of stressinduced ischemia and the poststress ejection fraction. $\mathrm{J}$ Nucl Med 2001;42:831-7.

[2] Thomas GS, Miyamoto MI, Morello 3rd AP, Majmundar H, Thomas JJ, Sampson CH, et al. Technetium 99m sestamibi myocardial perfusion imaging predicts clinical outcome in the community outpatient setting. The Nuclear Utility in the Community (NUC) Study. J Am Coll Cardiol 2004;43:213-23.

[3] Sharir T, Germano G, Kavanagh PB, Lai S, Cohen I, Lewin HC, et al. Incremental prognostic value of post-stress left ventricular ejection fraction and volume by gated myocardial perfusion single photon emission computed tomography. Circulation 1999;100:1035-42.

[4] White HD, Norris RM, Brown MA, Brandt PW, Whitlock RM, Wild CJ. Left ventricular end-systolic volume as the major determinant of survival after recovery from myocardial infarction. Circulation 1987;76:44-51.

[5] Bestetti A, Triulzi A, Di Leo C, Tagliabue L, Strinchini A, Bax J. Enhanced prognostic stratification of CAD patients with low ejection fraction by stressrest Tc99m tetrofosmin gated-spect. Acta Cardiol 2004;59:17-23.

[6] Mowatt G, Vale L, Brazzelli M, Hernandez R, Murray A, Scott N, et al. Systematic review of the effectiveness and cost-effectiveness, and economic evaluation, of myocardial perfusion scintigraphy for the diagnosis and management of angina and myocardial infarction. Health Technol Assess 2004;8:1-207.

[7] Kayano D, Nakajima K, Ohtake H, Kinuya S. Gated myocardial perfusion SPECT for preoperative risk stratification in patients with noncardiac vascular disease. Ann Nucl Med 2009;23:173-81.

[8] Kapetanopoulos A, Ahlberg AW, Taub CC, Katten DM, Heller GV. Regional wall-motion abnormalities on post-stress electrocardiographic-gated technetium-99m sestamibi single-photon emission computed tomography imaging predict cardiac events. J Nucl Cardiol 2007;14:810-7.

[9] Petix NR, Sestini S, Coppola A, Marcucci G, Nassi F, Taiti A, et al. Prognostic value of combined perfusion and function by stress technetium-99m sestamibi gated SPECT myocardial perfusion imaging in patients with suspected or known coronary artery disease. Am J Cardiol 2005;95:1351-7

[10] Swaminathan M, Morris RW, De Meyts DD, Podgoreanu MV, Jollis JG, Grocott HP, et al. Deterioration of regional wall motion immediately after coronary artery bypass graft surgery is associated with long-term major adverse cardiac events. Anesthesiology 2007;107:739-45.

[11] Spinelli L, Petretta M, Acampa W, He W, Petretta A, Bonaduce D, et al. Prognostic value of combined assessment of regional left ventricular function and myocardial perfusion by dobutamine and rest gated SPECT in patients with uncomplicated acute myocardial infarction. J Nucl Med 2003;44:1023-9.

[12] Travin MI, Heller GV, Johnson LL, Katten D, Ahlberg AW, Isasi CR, et al. The prognostic value of ECG-gated SPECT imaging in patients undergoing stress Tc-99m sestamibi myocardial perfusion imaging. J Nucl Cardiol 2004;11:253-62.

[13] Fleischmann S, Koepfli P, Namdar M, Wyss CA, Jenni R, Kaufmann PA. Gated (99m)Tc-tetrofosmin SPECT for discriminating infarct from artifact in fixed myocardial perfusion defects. J Nucl Med 2004;45:754-9.

[14] Miles KA. How does gated SPET alter reporting of myocardial perfusion studies? Nucl Med Commun 1997;18:915-21.

[15] Sharir T, Bacher-Stier C, Dhar S, Lewin HC, Miranda R, Friedman JD, et al. Identification of severe and extensive coronary artery disease by postexercise regional wall motion abnormalities in Tc-99m sestamibi gated single-photon emission computed tomography. Am J Cardiol 2000;86:1171-5.

[16] Emmett L, Iwanochko RM, Freeman MR, Barolet A, Lee DS, Husain M. Reversible regional wall motion abnormalities on exercise technetium99m-gated cardiac single photon emission computed tomography predict high-grade angiographic stenoses. J Am Coll Cardiol 2002;39:991-8.

[17] Levine MG, McGill CC, Ahlberg AW, White MP, Giri S, Shareef B, et al. Functional assessment with electrocardiographic gated single-photon emission computed tomography improves the ability of technetium- $99 \mathrm{~m}$ sestamibi myocardial perfusion imaging to predict myocardial viability in patients undergoing revascularization. Am J Cardiol 1999;83:1-5.

[18] Duncan BH, Ahlberg AW, Levine MG, McGill CC, Mann A, White MP, et al. Comparison of electrocardiographic-gated technetium-99m sestamibi single-photon emission computed tomographic imaging and restredistribution thallium-201 in the prediction of myocardial viability. Am J Cardiol 2000;85:680-4.

[19] Germano G, Kavanagh PB, Su HT, Mazzanti M, Kiat H, Hachamovitch R, et al. Automatic reorientation of three-dimensional, transaxial myocardial perfusion SPECT images. J Nucl Med 1995;36:1107-14.

[20] Germano G, Kiat H, Kavanagh PB, Moriel M, Mazzanti M, Su HT, et al. Automatic quantification of ejection fraction from gated myocardial perfusion SPECT. J Nucl Med 1995;36:2138-47.

[21] Germano G, Kavanagh PB, Waechter P, Areeda J, Van Kriekinge S, Sharir $\mathrm{T}$, et al. A new algorithm for the quantitation of myocardial perfusion SPECT. I: technical principles and reproducibility. J Nucl Med 2000;41:712-9.

[22] Germano G, Kavanagh PB, Slomka PJ, Van Kriekinge SD, Pollard G, Berman DS. Quantitation in gated perfusion SPECT imaging: the CedarsSinai approach. J Nucl Cardiol 2007;14:433-54.

[23] Nichols KJ, Van Tosh A, Wang Y, Chen J, Garcia EV, Palestro CJ, et al. Relationships between blood pool and myocardial perfusion gated SPECT global and regional left ventricular function measurements. Nucl Med Commun 2009;30:292-9.

[24] Nichols KJ, Van Tosh A, Wang Y, Palestro CJ, Reichek N. Validation of gated blood-pool SPECT regional left ventricular function measurements. J Nucl Med 2009;50:53-60.

[25] Mariano-Goulart D, Collet H, Kotzki PO, Zanca M, Rossi M. Semiautomatic segmentation of gated blood pool emission tomographic images by watersheds: application to the determination of right and left ejection fractions. Eur J Nucl Med 1998;25:1300-7.

[26] Caderas de Kerleau C, Crouzet JF, Ahronovitz E, Rossi M, MarianoGoulart D. Automatic generation of noise-free time-activity curve with gated blood pool emission tomography using deformation of a reference curve. IEEE Trans Med Imaging 2004;23:485-91.

[27] Cwajg E, Cwajg J, Keng F, He ZX, Nagueh S, Verani MS. Comparison of global and regional left ventricular function assessed by gated-SPECT and 2-D echocardiography. Rev Port Cardiol 2000;19(Suppl. 1):139-46.

[28] Chua T, Kiat H, Germano G, MAurer G, Friedman J, Berman D. Gated technetium-99m sestamibi for simultaneous assessment of stress 
myocardial perfusion, postexercise regional ventricular function and myocardial viability. Correlation with echocardiography and rest thallium-201 scintigraphy. J Am Coll Cardiol 1994;23:1107-14.

[29] Demir H, Tan YZ, Kozdag G, Isgoren S, Anik Y, Ural D, et al. Comparison of gated SPECT, echocardiography and cardiac magnetic resonance imaging for the assessment of left ventricular ejection fraction and volumes. Ann Saudi Med 2007;27:415-20

[30] Bax JJ, Lamb H, Dibbets P, Pelikan H, Boersma E, Viergever EP, et al Comparison of gated single-photon emission computed tomography with magnetic resonance imaging for evaluation of left ventricular function in ischemic cardiomyopathy. Am J Cardiol 2000;86:1299-305.

[31] Sibille L, Bouallegue FB, Bourdon A, Micheau A, Vernhet-Kovacsik H, Mariano-Goulart D. Comparative values of gated blood-pool SPECT and CMR for ejection fraction and volume estimation. Nucl Med Commun 2011;32:121-8.

[32] Williams KA, Taillon LA. Left ventricular function in patients with coronary artery diseasea assessed by gated tomographic myocardial perfusion images: comparison with assessment by contrast ventriculography and first-pass radionuclide angiography. J Am Coll Cardiol 1996;27:173-81.

[33] Vanhove C, Walgraeve N, De Geeter F, Franken PR. Gated myocardial perfusion tomography versus gated blood pool tomography for the calculation of left ventricular volumes and ejection fraction. Eur J Nucl Med Mol Imaging 2002;29:735-41.

[34] Cochet H, Bullier E, Gerbaud E, Durieux M, Godbert Y, Lederlin M, et al Absolute quantification of left ventricular global and regional function at nuclear MPI using ultrafast CZT SPECT: initial validation versus cardiac MR. J Nucl Med 2013;54:556-63.

[35] Levy M, Bekri N, Pouillart F, Bellorini M, Romano M, Perez T, et al. Comparison of segmental wall motion in electrocardiogram-gated myocardial scintigraphy and transthoracic echocardiography. Arch Mal Coeur Vaiss 2000;93:827-34.

[36] Hansen CL, Goldstein RA, Akinboboye OO, Berman DS, Botvinick EH, Churchwell KB, et al. Myocardial perfusion and function: single photon emission computed tomography. J Nucl Cardiol 2007;14:e39-60.

[37] Hesse B, Lindhardt TB, Acampa W, Anagnostopoulos C, Ballinger J, Bax $\mathrm{JJ}$, et al. EANM/ESC guidelines for radionuclide imaging of cardiac function. Eur J Nucl Med Mol Imaging 2008;35:851-85.

[38] Jaszczak RJ, Floyd Jr CE, Coleman RE. Scatter compensation techniques for SPECT. IEEE Trans Nucl Sci 1985;32:786-93.

[39] Sibille L, Bouallegue FB, Bourdon A, Mariano-Goulart D. Influence of CT-based attenuation correction in assessment of left and right ventricular functions with count-based gated blood-pool SPECT. J Nucl Cardiol 2011;18:1642-9.
[40] Daou D, Harel F, Helal BO, Fourme T, Colin P, Lebtahi R, et al. Electrocardiographically gated blood-pool SPECT and left ventricular function: comparative value of 3 methods for ejection fraction and volume estimation. J Nucl Med 2001;42:1043-9.

[41] Vilain D, Daou D, Casset-Senon D, Faraggi M, Le Guludec D. Optimal 3dimensional method for right and left ventricular Fourier phase analysis in electrocardiography-gated blood-pool SPECT. J Nucl Cardiol 2001;8 371-8.

[42] Mariano-Goulart D, Dechaux L, Rouzet F, Barbotte E, Caderas de Kerleau $\mathrm{C}$, et al. Diagnosis of diffuse and localized arrhythmogenic right ventricular dysplasia by gated blood-pool SPECT. J Nucl Med 2007;48:141623.

[43] Hesse B, Tägil K, Cuocolo A, Anagnostopoulos C, Bardiés M, Bax J, et al. EANM/ESC procedural guidelines for myocardial perfusion imaging in nuclear cardiology. Eur J Nucl Med Mol Imaging 2005;32:855-97.

[44] Nichols KJ, Van Tosh A, De Bondt P, Bergmann SR, Palestro CJ, Reichek N. Normal limits of gated blood pool SPECT count-based regional cardiac function parameters. Int J Cardiovasc Imaging 2008;24:717-25.

[45] Sharir T, Berman DS, Waechter PB, Areeda J, Kavanagh PB, Gerlach J, et al. Quantitative analysis of regional motion and thickening by gated myocardial perfusion SPECT: normal heterogeneity and criteria for abnormality. J Nucl Med 2001;42:1630-8.

[46] Bogaert J, Rademakers FE. Regional nonuniformity of normal adult human left ventricle. Am J Physiol Heart Circ Physiol 2001;280: H610-20

[47] Prasad M, Ramesh A, Kavanagh P, Tamarappoo BK, Nakazato R, Gerlach $\mathrm{J}$, et al. Quantification of 3D regional myocardial wall thickening from gated magnetic resonance images. J Magn Reson Imaging 2010;31:31727.

[48] Akhter N, Nakajima K, Okuda K, Matsuo S, Yoneyama T, Taki J, et al. Regional wall thickening in gated myocardial perfusion SPECT in a Japanese population: effect of sex, radiotracer, rotation angles and frame rates. Eur J Nucl Med Mol Imaging 2008;35:1608-15.

[49] Faber TL, Vansant JP, Pettigrew RI, Galt JR, Blais M, Chatzimavroudis G, et al. Evaluation of left ventricular endocardial volumes and ejection fractions computed from gated perfusion SPECT with magnetic resonance imaging: comparison of two methods. J Nucl Cardiol 2001;8:645-51.

[50] DePuey EG, Parmett S, Ghesani M, Rozanski A, Nichols K, Salensky H. Comparison of Tc-99m sestamibi and Tl-201 gated perfusion SPECT. J Nucl Cardiol 1999;6:278-85.

[51] America YG, Bax JJ, Dibbets-Schneider P, Pauwels EK, Van der Wall EE. Evaluation of the Quantitative Gated SPECT (QGS) software program in the presence of large perfusion defects. Int J Cardiovasc Imaging 2005;21: 519-29. 